\title{
Comparative Evaluation of Dental Anxiety and Fear in Children by using Camouflaged Syringe and Conventional Syringe
}

\author{
Monika K*, Poonacha KS, Anshula D, Bhavna D, Rameshwari R and \\ Neha $\mathbf{P}$ \\ Department of Pedodontics and Preventive Dentistry, K M Shah Dental College and \\ Hospital, India
}

\section{Research Article \\ Volume 4 Issue 1}

Received Date: January 10, 2019

Published Date: January 18, 2019

DOI: $10.23880 /$ oajds-16000214

*Corresponding author: Monika Khoja, Department of Pedodontics and Preventive Dentistry, K M Shah Dental College and Hospital, Vadodara, Gujarat, India, Tel: +919429299994; Email: monikakhoja@gmail.com

\section{Abstract}

Introduction: Dental Fear (DF) and Dental Anxiety (DA) are the terms, used to symbolize early signs of Dental Phobia (DP). Dental anxiety in children due to sight of injection is the most common. Local anesthesia technique using camouflaged syringes has been tried to decrease anxiety and fear in children. This study was conducted by using most reliable scale is Venham Picture Test (VPT) and Face, Leg, Activity, Cry and Constability (FLACC) Behavioral Pain Assessment Scale to assess anxiety and fear in different groups of children being administered local anesthetic (LA) using either conventional or camouflaged syringe.

Aim: To evaluate and compare dental anxiety and fear in children by using camouflaged and conventional syringe.

Methodology: Total 80 children aged between 6-8 years were included. The appearance of Camouflage Syringe was child-friendly. Pictures of smiles, cars, cartoon characters, colourful fishes and sun were integrated in the design of Camouflage Syringe. The trial had two groups- Camouflaged (CaS) and conventional syringe (CoS) group. Inferior alveolar nerve block was administered in both the groups using the allocated type of syringes. VPT and FLACC scales were used.

Results: A total of 52 male and 28 female children were integrated in the study. Comparison of the Venham picture test score and FLACC score in terms of [Mean (SD)] (SD - standard deviation) was done amongst both the groups using unpaired t-test. The FLACC score [Mean (SD)] for group I and II were, $2.53(0.5)$ and $1.48(0.5)$ respectively. The [Mean (SD)] for group I and Group II of VPT was 6.40 (1.2) and 3.85 (1.0) respectively, while that for FLACC was .53 (0.5) and $1.48(0.5)$ respectively.

Conclusion: The anxiety level of Female children was significantly higher than male children. By using Camouflaged Syringe, Fear and anxiety were significantly reduced.

Keywords: Fear; Anxiety; Camouflaged Syringe; Conventional Syringe 


\section{Open Access Journal of Dental Sciences}

Abbreviations: DF: Dental Fear; DA: Dental Anxiety; DP: Dental Phobia; VPT: Venham Picture Test; FLACC: Face, Leg, Activity, Cry and Constability; LA: Local Anesthetic; CaS: Camouflaged; CoS: Conventional Syringe.

\section{Introduction}

In Paediatric dentistry the success of treatment depends upon control of patients behavior and dentistpatient-parents relationship. One of the most tricky aspect of dental care for dental practitioners is administration of LA [1].

The term "injection", gives the child unpleasant feeling because of pain caused due to the needle prick and makes the child patient more fearful and uncooperative. Dental anxiety is more in paediatric patients which may be due to past painful medical and dental experiences and thus can also result in poor cognitive development of a child [2-4]. Even after such improved dental skills and mechanics, parents and paediatric patients still show negligence and hesitation towards dental treatment because of past dreadful experiences [2].

Various non-pharmacological behavior techniques are used by paediatric dentists to reduce the discomfort and anxiety level of children and thus, child's cooperation may increase while administering LA. Therefore, to minimize fear and anxiety, the knowledge of psychology and child behavior management techniques are essential for the dentist to establish a relationship of harmony and trust with patients and their families [4,5]. Anxiolytic drugs, mild sedation and general anesthesia have been considered for overcoming the therapeutic challenges posed by dental fear and anxiety in children. 6

The dictionary meaning of camouflage is to hide or disguise the presence of (a person, object, etc). Camouflaging of the syringe can be done to alleviate the child's anxiety and therefore it can demonstrate improved outcomes related to fear and anxiety $[6,7]$.

The child friendly look or the appearance of camouflage syringe can reduce the dental anxiety and fear and at the same time provides positive dental attitude. Even the camouflage syringe helps in distracting the patients while administering LA. It can help in providing the better treatment and treatment outcome [8].

By this, more child friendly environment can be created and fear and anxiety can be reduced. By effective anesthesia administration, the dental procedures like restorations, extractions, pulpotomies, pulpectomies and other minor surgical procedures which require the administration of LA specially the inferior alveolar block, can be made pleasant for the child. It will also help the child to improve his or her mental and psychological status. It can lead the child in the paediatric dental setup for further dental treatment without fear of injection.

\section{Aim}

To evaluate and compare dental anxiety and fear in children by using Camouflaged Syringe and Conventional Syringe.

\section{Methodology}

Approval was taken from the Institutional ethical committee of KMSDCH, Vadodara. Healthy children who did not have any previous dental experience were included. In the first dental visit children who fall under 2 and 3 Frankel's behaviour rating scale were included. Mandibular primary molars indicated for any dental procedure under inferior alveolar nerve block were considered in study. Mentally challenged, patients who were allergic to Lignocaine Local anesthetic solution, parents and individual's who were unwilling to give consent and accent respectively and Children with medical problems that negate the use of LA and/or surgical procedures were excluded from the study. A clinical assistant was trained to assist in documenting and recording the observation done by the investigator in the study.

Disposable syringe was used. (NIPRO SYRINGE with needle, $2.5 \mathrm{ml})$ Needle $=27 \mathrm{G}^{*} 1.5 "\left(0.4^{*} 38 \mathrm{~mm}\right)$ The appearance of Camouflage Syringe cover was attractive and child-friendly, it was hand made using cold cure acralylic and included pictures of smileys, cars, cartoon characters, colourful fishes and sun. Cover consist of three parts: Head, Shank and Tail. The head holds the needle and the proximal part of the normally used conventional syringe and has a viewing window to check for aspiration results. The tail holds the rest of the normal syringe loaded with LA solution along with the plunger. Children were equally divided into two groups. Camouflaged syringe grouped children were given choice to select the camouflaged cover. In the case of Camouflage Syringe, conventional syringe was filled with anesthetic solution out of the site of the patient and then it was inserted into Camouflage cover. The local anesthetic gel was applied and inferior alveolar nerve block was given by conventional technique in both the groups and FLACC 


\section{Open Access Journal of Dental Sciences}

Behavioural pain score was recorded. And after administration of local anesthesia VPT score (pictures were shown to participants) was recorded.

\section{Statistical Analysis}

Descriptive and inferential statistical analysis were carried out in the study. Results on continuous measurements were presented on [Mean (SD)] and results on categorical measurements were presented in Number (\%). Level of significance was fixed at $\mathrm{p}=0.05$ and any value less than or equal to 0.05 was considered to be statistically significant.

a) Chi square analysis was used to find the significance of study parameters on categorical scale.

b) Student ' $t$ ' test (two tailed, independent) was used to find the significance of study parameters on continuous scale between two groups (Inter group analysis) on metric parameters.

\section{Result}

80 children were equally divided into two groups.

\begin{tabular}{|c|c|c|}
\hline Variables & Sub-groups & n (\%) \\
\hline \multirow{2}{*}{ Group } & Conventional syringe & $40(50)$ \\
\cline { 2 - 3 } & Camouflaged syringe & $40(50)$ \\
\hline \multirow{2}{*}{ Gender } & Male & $52(65)$ \\
\cline { 2 - 3 } & Female & $28(35)$ \\
\hline \multirow{3}{*}{ Age (Years) } & 6 & $30(37.5)$ \\
\cline { 2 - 3 } & 7 & $23(28.7)$ \\
\cline { 2 - 3 } & 8 & $27(33.8)$ \\
\hline
\end{tabular}

Table 1: Demographic characteristics of the study participants $(\mathrm{N}=80)$.

There were $52(n=65 \%)$ male participants and $28(n=$ $35 \%)$ female participants.

\begin{tabular}{|c|c|c|}
\hline Group & No of samples & Mean (SD) \\
\hline Conventional syringe & 40 & $6.40(1.2)$ \\
\hline Camouflaged syringe & 40 & $3.85(1.0)$ \\
\hline t value & - & 9.75 \\
\hline P value & - & $<0.001^{* *}$ \\
\hline
\end{tabular}

( $\mathrm{p}<0.05$ - Significant*, $\mathrm{p}<0.001$ - Highly significant**)

Table 2: Comparison of the Venham picture test score in terms of [Mean (SD)] among both the groups using unpaired $t$ test.
Here, $p$ value is $<0.001$, which is statistically highly significant. This shows obvious reduction in fear and anxiety in children on whom camouflaged syringe was used.

\begin{tabular}{|c|c|c|}
\hline Group & No of samples & Mean (SD) \\
\hline $\begin{array}{c}\text { Conventional } \\
\text { syringe }\end{array}$ & 40 & $2.53(0.5)$ \\
\hline $\begin{array}{c}\text { Camouflaged } \\
\text { syringe }\end{array}$ & 40 & $1.48(0.5)$ \\
\hline t value & - & 8.141 \\
\hline P value & - & $<0.001^{* *}$ \\
\hline
\end{tabular}

Table 3: Comparison of the FLACC score in terms of [Mean (SD)] among both the groups using unpaired $t$ test.

Here, $p$ value is $<0.001$, which is highly significant. [Mean (SD)] are $2.53(0.5)$ and 1.48 (0.5), for Conventional syringe and Camouflaged syringe respectively.

\section{Discussion}

Despite great progress in paediatric dentistry, many children require dental treatment of various forms. Handmade Camouflage Syringes using cold cure acrylic was made. However, more robust and long-lasting syringes can be designed using polyethylene materials like polyvinyl chloride which are more suitable to mass production. The used material is easily available in dental clinic. The camouflaged cover used on the syringe was given as a gift to participants, this had acted as a positive reinforcement. In this study, we have observed that a simple and novel innovation in the appearance of the conventional syringe can result in significantly improved outcomes related to dental fear and anxiety. The primary as well as secondary outcomes showed increased compliance of children. It is remarkable that the Camouflage Syringe naturally permits more involvement of patient choices into the treatment protocol, thus helping to alleviate dental fears. We did not observe any local infections in either group; therefore a direct comparison of the surface contamination rates could not be done. Similar study was done by S. Ujaoney et al8 in 2013 , they had conducted a randomized controlled trial study in 100 children aged $<15$ years of age. They concluded that the Camouflage Syringe is highly efficacious in dental procedures requiring a fearcontrolled delivery of topical or local anaesthesia. Similar results were obtained from the present study. 


\section{Open Access Journal of Dental Sciences}

\section{Conclusion}

Within the limitation of the study, it was concluded that Female children were more anxious before treatment. By using camouflaged syringe, there was significant reduction in the anxiety and fear in children aged between 6-8 years, camouflaging is effective method to allay fear and anxiety. Reduction of fear and anxiety were more in female patients compared to males.

\section{References}

1. Cohen LA, Harris SL, Bonito AJ, Manski RJ, Macek MD, et al. (2007) Coping with toothache pain: a qualitative study of low-income persons and minorities. J Public Health Dent 67(1): 28-35.

2. Hodes RL, Howland EW, Lightfoot N, Cleeland CS (1990) The effects of distraction on responses to cold pressor pain. Pain 41(1): 109-114.

3. Sharar SR, Miller W, Patterson DR (2008) Applications of virtual reality for pain management in burn-injured patients. Expert Rev Neurother 8(11): 1667-1674.
4. Wiederhold BK, Wiederhold MD (2012) Managing pain in military populations with virtual reality. NATO Science for Peace \& Security Series-E: Human \& Societal Dynamics 91: 75-93.

5. Elledge R, Alexopoulos E, Hosey MT (2007) Short communication: dental anxiety levels and outcomes of care: a preliminary report on experiences of a sedation assessment clinic. Eur Arch Paediatr Dent 8(4): 211-214.

6. Klinber G (2008) Dental anxiety and behaviour management problems in paediatric dentistry-a review of background factors and diagnostics. Eur Arch Paediatr Dent 9(1): 11-15.

7. Bellini M, Maltoni O, Gatto MR, Pelliccioni G, Checchi $\mathrm{V}$, et al. (2008) Dental phobia in dentistry patients. Minerva Stomatol 57(10): 485-495.

8. Ujaoney S, Mamtani M, Thakre T, Tote J, Hazarey V, et al. (2013) Efficacy trial of Camouflage Syringe to reduce dental fear and anxiety. EJPD 14(4): 273-278. 